\title{
The Mediating Role of Pain Catastrophizing on the Association Between Depression and Pain Severity and Interference Among Elderly Asian Immigrants with Chronic Pain
}

This article was published in the following Dove Press journal:

Journal of Pain Research

\author{
Hee Jun Kim' \\ Hyunjeong Park $\mathbb{D}^{2}$ \\ Hee-Soon Juon ${ }^{3}$ \\ 'Research Institute of Nursing Science, \\ College of Nursing, Ajou University, \\ Suwon, 16499, Republic of Korea; \\ ${ }^{2}$ Department of Nursing, Towson \\ University, Towson, MD, 21252, USA; \\ ${ }^{3}$ Department of Medical Oncology, \\ Thomas Jefferson University, Philadelphia, \\ PA, 19107, USA
}

Correspondence: Hee Jun Kim Research Institute of Nursing Science, College of Nursing, Ajou University, 206

World cup-ro, Yeongtong-gu, Suwon-si,

Gyeonggi-do, Republic of Korea

Tel +82 3I-2I9-7024

Fax +82 3I-219-7020

Email hkim20@ajou.ac.kr
Purpose: The association between depression and chronic pain is well established. However, few studies have examined the pathways from depression to chronic pain. The present cross-cultural study aimed to test the mediating effects of pain catastrophizing on associations between depression and chronic pain (eg, pain severity, pain intensity) among Korean American elderly.

Patients and Methods: A total of 132 elderly Korean Americans with chronic pain were recruited from elderly daycare centers and Korean ethnic churches in the community. For mediation analyses, structural equation modeling with full information maximum likelihood estimation method was used. The bias-corrected bootstrap confidence interval (CI) method for inferential tests of the indirect effects was also conducted in mediation analysis.

Results: The results indicated that the proportion of comorbid depression and chronic pain was $45.7 \%$. Elderly Korean Americans with depression scored higher in pain severity, pain interference, and pain catastrophizing than those without depression. Pain catastrophizing was found to have a significant mediating effect on the relationship between depression and pain severity (indirect effect $=0.147$, Bootstrap 95\% CI $=[0.079,0.226]$ ), controlling for demographic covariates, comorbidities and pain area. Pain catastrophizing also mediated the relationship between depression and pain interference (indirect effect $=0.164$, Bootstrap 95\% $\mathrm{CI}=[0.097,0.244])$, controlling for the covariates.

Conclusion: These findings add to the literature by providing evidence that pain catastrophizing plays a role in high levels of pain severity and pain interference comorbid chronic pain and depression in Asian elderly immigrants. Appropriate culturally tailored programs to redirect pain catastrophizing cognitive process should be developed and provided for elderly Asian Americans to reduce chronic pain disparity.

Keywords: Korean immigrants, cross-cultural, comorbid, mediation

\section{Introduction}

Chronic pain, which is pain associated with direct or potential tissue injury and lasting or recurring for more than three months, is characterized as subjective, involving both sensory and emotional responses. ${ }^{1}$ Chronic pain is considered a major health concern, with negative effects that include low quality of life, psychosocial distress, pain-induced disability, and opioid abuse or overdose. ${ }^{2-4}$ Among immigrants, the experience of chronic pain may be influenced by several 
factors, including a lack of social/emotional support, different sociocultural environment, and language barrier., Immigrants' pain experience and pain-related disease progression may also be affected by cultural beliefs related to pain and by the different pain management systems used in different healthcare environments. For example, Asian immigrants consider pain part of the normal aging process and furthermore accept pain and suffering for spiritual growth. ${ }^{4}$ Asian immigrants were reported to have greater pain tolerance compared to non-Hispanic whites, ${ }^{7}$ are reluctant to report pain, and have less tendency to complain because they do not want to burden others. ${ }^{4}$ Language barriers, different health insurance system, and lack of preferable alternative pain management methods (eg, Chinese Traditional Medicine) may also influence chronic pain experience among elderly immigrants. ${ }^{8}$ A previous study on intra-racial differences in chronic pain severity in the elderly found a higher level of chronic pain severity among Korean immigrants compared to their counterparts in their home country. ${ }^{9}$ Years of living in the US may vary among the immigrant participants, however, their acculturation level was low - the immigrant group preferred Korean language, music, and food. ${ }^{9}$

Chronic pain is often associated with depression, with comorbidity rates ranging from $30 \%$ to $60 \%$ and disease progression that is influenced by each other. ${ }^{10,11}$ Depression can exacerbate chronic pain progression and reduce analgesic effects of pain medication. ${ }^{12}$ Among Asian Americans, the prevalence of depression has been reported to be $13-45 \%$, depending upon how it is measured and cut-offs for depression diagnosis. ${ }^{13}$ This high prevalence of depression may influence the experience of chronic pain and disease progression among Asian Americans.

Pain catastrophizing is exacerbated negative thinking in response to pain, and includes helplessness, rumination, and magnification related to the cognitive and emotional pain experience. Patients with pain catastrophizing have a tendency to overestimate the threat from painful sensations, and pain catastrophizing, as a maladaptive response to pain, may be associated with other psychological distress, including depression. ${ }^{14}$ Patients with depression may have increased negative emotional responses to their pain (eg, helplessness, hopelessness), which can evoke pain catastrophizing thoughts. A higher level of pain catastrophizing has been reported among Asian Americans, compared to non-Hispanic whites, and this has been associated with a higher sensitivity to pain in Asian Americans. ${ }^{7}$
Previously, depression, pain catastrophizing, and pain severity/pain interference have been studied widely; however, most studies examined depression as an outcome of interest or mediator. For example, a previous report of depression mediating the association between pain catastrophizing and pain-related factors, including pain intensity among elderly. ${ }^{15}$ Pain catastrophizing mediates the associations between pain intensity and depression. ${ }^{16-18}$

The possible mechanisms for how depression influences the chronic pain experience have not been examined and need to be explored given the high prevalence of depression $^{13}$ and chronic pain severity ${ }^{9}$ in this particular population. The biopsychosocial framework of pain emphasizes multidimensional aspects of pain, including psychological dysfunction (eg, depression), social factors (eg, income, education, immigrant status), and biological factors (eg, gender, comorbidity). A multidimensional approach to manage chronic pain among the elderly population has been emphasized to provide effective and personalized care to improve pain-related outcomes. ${ }^{19}$ The purpose of the present study was to test the mediating effect of pain catastrophizing on depression, pain severity, and pain intensity among Korean elderly immigrants with chronic pain in the United States. We hypothesized that: 1) individuals with comorbid chronic pain and depression will have higher levels of pain severity and pain interference compared to those without depression, and 2) the association between depression and pain severity and pain interference will be mediated by pain catastrophizing.

\section{Patients and Methods}

\section{Participants}

This study was part of a cross-cultural comparison study on chronic pain between Korean-Americans living in the United States and Koreans living in their home country. The parent study was approved by the Towson University's Institutional Review Board for KoreanAmerican study site. This study was in accordance with the Declaration of Helsinki. Informed consent was obtained from all participants and information about the study was provided prior to that, including aims, methods, potential benefits and risks of the study, contact information of the researchers, confidentiality of the data and anonymity of their participation. Eligibility criteria testing and data collection were conducted in elderly daycare centers and Korean churches by three bilingual (Korean and English) registered nurses. All questionnaires were 
Korean versions of the measures, and the registered nurses answered any questions raised by the participants and reviewed measures after completion to minimize missing data. The participants were $\geq 65$ years and had selfreported chronic musculoskeletal pain for more than 3 months. Participants with cognitive impairment (MiniMental Status Examination <23/30) were excluded from the study, and other specific inclusion/exclusion criteria are described elsewhere. ${ }^{9}$ A total of 132 Korean Americans voluntarily consented to participate in the study.

\section{Measures}

Demographic characteristics included in the study were age, gender, educational status, perceived socio-economic status, and marital status. Self-reported comorbidities were assessed for commonly prevalent disease in elderly, including hypertension, arthritis, osteoporosis, diabetes, and others (eg, cancer, heart disease, cerebrovascular disease, problems related to prostate, urinary track, eye, or ear).

\section{Pain Severity and Interference}

The Brief Pain Inventory (BPI), which has been validated in the Korean population previously, was used to measure pain severity and pain interference. ${ }^{20,21}$ Pain areas were assessed by asking participants shade on the diagram (front and back of the body) in the areas where they feel pain. For pain severity, the average of four items of BPI measuring pain severity (now, at its worst, least, and average over the past week) were used. The pain interference score was calculated by averaging nine items evaluating the degree of interferences due to pain with general activity, mood, walking, work, sleep, relations with others, and enjoyment of life. Cronbach's alpha for pain severity and pain interference were 0.86 and 0.91 , respectively, in the current study.

\section{Depressive Symptoms}

Depressive symptoms were measured using the Patient Health Questionnaire-9 (PHQ-9) Korean version. ${ }^{22}$ The PHQ-9 is a self-administered questionnaire for depression screening and diagnosis. The nine items included in the measurement are based on the 9 diagnostic criteria for a major depressive episode in Diagnostic and Statistical Manual of Mental Disorders (DSM-5) ${ }^{23}$ Higher scores on the PHQ-9 are indicative of higher depressive symptoms. When the participants scored 5 or above on the
PHQ-9, they were considered to have depression. The validity and reliability of PHQ-9 have been reported for the Korean population previously, ${ }^{24}$ and the Cronbach's alpha for the current study was 0.80 .

\section{Pain Catastrophizing}

The Pain Catastrophizing Scale (PCS) was used to measure pain catastrophizing. ${ }^{25}$ The total sum of 13-item responses provides a good index of the catastrophizing construct through the highly correlated subscales of rumination, helplessness, and magnification. Higher scores on the PCS are indicative of greater pain-related catastrophizing. The validity of this scale has been reported for Asian Americans, ${ }^{26}$ and the Korean version of the PCS (K-PCS), which has been validated previously, was used for this study. ${ }^{27}$ The Cronbach's alpha was 0.94 for this study.

\section{Statistical Analyses}

To describe the demographic characteristics of the participants, frequency, mean, range, and standard deviation were presented. To test the difference in pain severity, pain interference, and pain catastrophizing between participants with depression and without depression, independent groups $t$-test was conducted. Preliminary analyses were conducted to check the assumptions of normality, linearity, homoscedasticity, and multicollinearity. Bivariate associations among the study variables were tested using Pearson correlation. For mediation analyses, we used structural equation modeling using the full information maximum likelihood estimation method. Two separate models for pain severity and pain interference were analyzed. Demographic characteristics that showed significant association in bivariate analyses with the outcome variable were included in the multiple mediation analysis to control confounding variables. We also conducted the bias-corrected bootstrap confidence interval (CI) method for inferential tests of the indirect effects in mediation analysis. ${ }^{28}$ Five thousand bootstrap iterations created the bootstrap sample for estimating the indirect effect of mediation analysis. If a bootstrap $95 \% \mathrm{CI}$ includes 0 , then the indirect effect is considered non-significant. Missing data were minimal (ranged from 0 to 7 out of 132) for all study variables. A $p<0.05$ (2-sided) was considered statistically significant. Statistical analyses were performed using STATA 15.1 (StataCorp, Texas, USA). 


\section{Results}

Sociodemographic Characteristics, Comorbid Conditions, and Reported Chronic Pain Among the Participants

Demographic characteristics for the participants $(n=132)$ are presented in Table 1. The mean age of participants was 78.3 years ( $\mathrm{SD}=6.68$, range 65 to 95 years), and approximately $80 \%$ of the participants were female. About $29 \%$ of the participants reported at least some college education, and $60 \%$ reported that their perceived economic status was not difficult at all for daily living. The most prevalent chronic disease was hypertension (53\%), followed by arthritis (44\%), osteoporosis (33\%), and diabetes (32\%). The most prevalent chronic pain area was back $(49 \%)$, followed by knee (38\%), shoulder (25\%), and legs $(21 \%)$.

\section{Differences in Pain Severity, Pain Interference, and Pain Catastrophizing Between Patients with Depression and without Depression}

The mean score of pain severity was 3.77 ( $\mathrm{SD}=2.18)$. The mean scores of pain interference were $2.55(\mathrm{SD}=2.01)$, and $15.61(\mathrm{SD}=11.47$ ) for pain catastrophizing. For depression, the mean was $5.48(\mathrm{SD}=5.35)$, with $45.7 \%$ crossing the cutoff of 5 indicating depression. Table 2 shows the group differences in pain severity, pain interference, and pain catastrophizing between patients with depression and without depression. Significant differences were found in pain severity, pain interference, and all subscales and total scores of pain catastrophizing between the two groups $(\mathrm{p}<0.001)$. Participants with depression scored higher in pain severity, pain interference, and pain catastrophizing than those without depression.

\section{Mediating Effect of Pain Catastrophizing Between Depression and Pain Severity/ Pain Intensity}

Demographic characteristics, including age, gender, education level, perceived economic status, and living with spouse, were significantly associated with pain severity and/or pain interference in bivariate analysis (Table 3). Total number of comorbidities (pain severity: $r=0.424$, $\mathrm{p}<0.001$; pain interference: $\mathrm{r}=0.366, \mathrm{p}<0.001$ ), and pain area (pain severity: $\mathrm{r}=0.255, \mathrm{p}=0.003$; pain interference: $\mathrm{r}=0.374, \mathrm{p}<0.001$ ) were also significantly associated with pain severity and pain interference.
Table I Sample Characteristics of Korean-American Elderly with Chronic Pain $(\mathrm{N}=132)$

\begin{tabular}{|c|c|}
\hline Characteristics & $\mathbf{N}(\%)$ \\
\hline Age (mean $\pm S D$, range) & $78.33 \pm 6.68,65-95$ \\
\hline \multicolumn{2}{|l|}{ Gender } \\
\hline Male & $24(18.18)$ \\
\hline Female & $108(81.82)$ \\
\hline \multicolumn{2}{|l|}{ Education } \\
\hline No education & $4(3.03)$ \\
\hline$\leq$ Elementary graduate & $21(15.91)$ \\
\hline$\leq$ Middle school graduate & $26(19.70)$ \\
\hline$\leq$ High school graduate & $43(32.58)$ \\
\hline$\leq$ Some college & 31 (23.48) \\
\hline$\geq$ Graduate school & $7(5.30)$ \\
\hline \multicolumn{2}{|l|}{ Perceived economic status } \\
\hline Somewhat difficult for daily living & $10(7.69)$ \\
\hline Very difficult for daily living & $42(32.31)$ \\
\hline Not difficult at all for daily living & $78(60.00)$ \\
\hline \multicolumn{2}{|l|}{ Living with spouse } \\
\hline Yes & $74(56.06)$ \\
\hline No & $58(43.94)$ \\
\hline \multicolumn{2}{|l|}{ Comorbidity (multiple responses) } \\
\hline Hypertension & $70(53.03)$ \\
\hline Arthritis & $58(43.94)$ \\
\hline Osteoporosis & $43(32.58)$ \\
\hline Diabetes & $42(31.82)$ \\
\hline Eye-related disease & $33(25.00)$ \\
\hline Incontinence \& bladder-related disease & $26(19.70)$ \\
\hline Heart disease & $22(16.67)$ \\
\hline No. of comorbid conditions, median (IQR) & $2(1-4)$ \\
\hline \multicolumn{2}{|l|}{$\begin{array}{l}\text { Types of chronic pain area (multiple } \\
\text { responses) }\end{array}$} \\
\hline Back & $64(48.48)$ \\
\hline Knee & $50(37.87)$ \\
\hline Shoulder & $33(25.00)$ \\
\hline Leg & $28(2 \mid .2 I)$ \\
\hline Arm (including elbow) & $20(15.15)$ \\
\hline Neck & $13(9.84)$ \\
\hline Finger (including wrist) & II (8.33) \\
\hline Foot (including ankle) & $9(6.81)$ \\
\hline Hip (including hip joint) & $7(5.30)$ \\
\hline Others (abdomen, chest, head area) & II (8.33) \\
\hline No. of pain areas, median (IQR) & $2(I-4)$ \\
\hline
\end{tabular}

Among the demographic variables, age, education, and perceived economic status were highly correlated with number of comorbidity (Table 3). Therefore, age, education, and perceived economic status were excluded in the model to avoid multicollinearity. Gender, living with 
Table 2 Differences in Pain Severity, Pain Interference, and Pain Catastrophizing by Depressive Symptoms ( $N=132)$

\begin{tabular}{|l|c|c|c|c|c|c|c|c|}
\hline & \multicolumn{2}{|c|}{ Participants without Depression } & \multicolumn{2}{c|}{ Participants with Depression } & \multicolumn{1}{c|}{} \\
\cline { 2 - 8 } & Mean & SD & Range & Mean & SD & Range & t & P \\
\hline Pain severity & 2.94 & 1.92 & $0-8.3$ & 4.59 & 2.01 & $0.5-9.0$ & -4.756 & $<0.001$ \\
Pain interference* & 1.63 & 1.62 & $0-6.6$ & 3.53 & 1.96 & $0.1-6.7$ & -5.932 & $<0.001$ \\
\hline Pain catastrophizing & & & & & & & & \\
$\quad$ Rumination & 5.11 & 4.63 & $0-16$ & 9.65 & 4.95 & $0-16$ & -5.343 & $<0.001$ \\
Magnification & 2.10 & 2.61 & $0-12$ & 4.50 & 3.41 & $0-9$ & -4.507 & $<0.001$ \\
Helplessness & 2.07 & 2.04 & $0-8$ & 9.34 & 3.74 & $4-11$ & -13.91 & $<0.001$ \\
Total & 9.26 & 7.90 & $0-34$ & 23.29 & 10.38 & $7-34$ & -8.607 & $<0.001$ \\
\hline
\end{tabular}

Table 3 Correlations Among the Study Variables $(\mathrm{N}=132)$

\begin{tabular}{|c|c|c|c|c|c|c|c|c|c|c|}
\hline & $\mathbf{I}$ & 2 & 3 & 4 & 5 & 6 & 7 & 8 & 9 & 10 \\
\hline I. Age & - & & & & & & & & & \\
\hline 2. Gender & $<0.001$ & - & & & & & & & & \\
\hline 3. Living with spouse & $0.183^{*}$ & $0.338^{* *}$ & - & & & & & & & \\
\hline 4. Education & $-0.268 * *$ & -0.134 & -0.057 & - & & & & & & \\
\hline 5. Perceived economic status & -0.145 & 0.016 & -0.006 & 0.167 & - & & & & & \\
\hline 6. No. of pain areas & 0.106 & 0.039 & -0.029 & -0.041 & $-0.197 *$ & - & & & & \\
\hline 7. No. of comorbidity & $0.304 * *$ & 0.089 & 0.023 & $-0.296 * *$ & $-0.302 * *$ & $0.184 *$ & - & & & \\
\hline 8. Depression & 0.165 & 0.062 & 0.082 & -0.169 & $-0.332 * *$ & 0.178 & 0.149 & - & & \\
\hline 9. Pain Catastrophizing & $0.193^{*}$ & 0.134 & 0.109 & -0.166 & $-0.256^{*}$ & $0.246 *$ & $0.244 *$ & $0.77 I^{* *}$ & - & \\
\hline 10. Pain Severity & $0.206 *$ & $0.207^{*}$ & $0.187^{*}$ & $-0.322 * *$ & $-0.295^{* *}$ & $0.366 * *$ & $0.424 * *$ & $0.443 * *$ & $0.614 * *$ & - \\
\hline II. Pain interference & $0.211 *$ & 0.135 & 0.082 & -0.113 & -0.127 & $0.374 * *$ & $0.255^{*}$ & $0.55 I^{* *}$ & $0.705^{* *}$ & $0.759 * *$ \\
\hline
\end{tabular}

Notes: $*_{p}<0.05, *_{p}<0.001$.

spouse, numbers of comorbidity and pain areas were all included in the multivariate model as covariates. To explore the mediation effects of pain catastrophizing on the associations of depression with pain severity and depression with pain interference, multiple mediation analyses were conducted. The results of the mediating effects of pain catastrophizing are presented in Table 4. A significant mediating effect was found for pain catastrophizing on the relationship between depressive symptoms and pain severity (indirect effect $=0.135$, Bootstrap 95\% $\mathrm{CI}=[0.068,0.211])$, controlling for the covariates (Figure 1A). Pain catastrophizing also mediated the

Table 4 Mediation Analysis on Relationship Between Depressive Symptoms and Pain Severity/Pain Interference

\begin{tabular}{|l|c|c|c|c|c|c|}
\hline \multirow{2}{*}{} & \multicolumn{3}{|c|}{ Pain Severity } & \multicolumn{3}{c|}{ Pain Interference } \\
\cline { 2 - 6 } & Coefficient & s.e. & P & Coefficient & s.e. & P \\
\hline Direct effect (Depressive symptoms) & -0.014 & 0.039 & 0.718 & 0.013 & 0.038 \\
\hline Indirect effect (via mediator) & & & & & \\
Pain Catastrophizing & 0.135 & 0.036 & $<0.001$ & 0.162 & 0.035 \\
\hline Covariates & & & & & \\
Gender & 0.256 & 0.388 & 0.511 & 0.001 \\
Living with spouse & 0.454 & 0.295 & 0.127 & 0.052 & 0.371 \\
No. of comorbidity & 1.049 & 0.285 & $<0.001$ & 0.283 & 0.825 \\
No. of pain area & 0.715 & 0.288 & 0.015 & 0.835 & 0.273 \\
\hline
\end{tabular}

Notes: Gender was coded as $0=$ male, I=female; Living with spouse coded as $0=y e s, I=$ no; no. of comorbidity was coded as $0=\leq 2$, $1>2$; no. of pain area was coded as $0=\leq 2$, $1 \leq 2$. 


\section{A Mediating effect of pain catastrophizing between depression and pain severity}

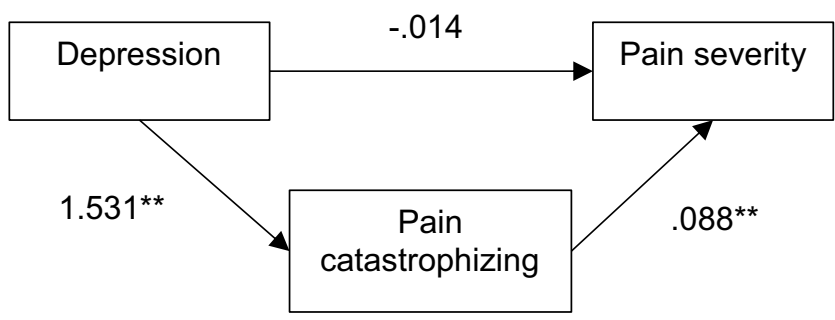

B Mediating effect of pain catastrophizing between depression and pain interference

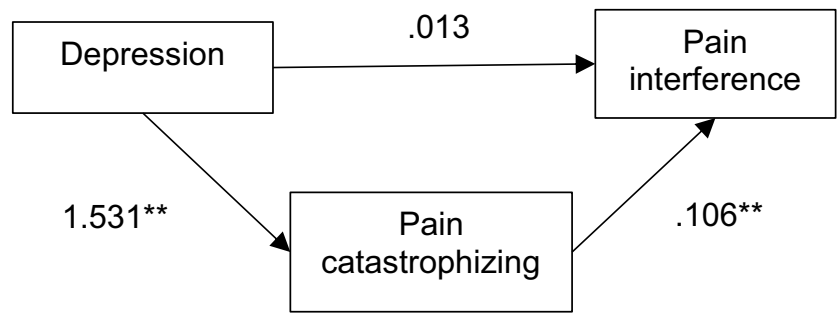

Figure I Mediational models. (A) Mediating effect of pain catastrophizing between depression and pain severity. (B) Mediating effect of pain catastrophizing between depression and pain interference.

Notes: Total effect $(0.121)=$ direct effect $(-0.014)+$ indirect effect $(1.531 \times 0.088)$ for $(\mathbf{A})$. Total effect $(0.175)=$ direct effect $(0.013)+$ indirect effect $(1.531 \times 0.106)$ for $(\mathbf{B})$ Covariates entered: gender, living with spouse, numbers of comorbidity and pain area; Just-identified model (no model fit indices); ${ }^{*} \mathrm{p}<0.0 \mathrm{I},{ }^{*} \mathrm{P}<0.00 \mathrm{I}$.

relationship between depressive symptoms and pain interference (indirect effect $=0.162$, Bootstrap 95\% CI $=$ $[0.097,0.236]$ ), controlling for the covariates (Figure 1B).

\section{Discussion}

The present study explored the associations between depression and pain severity and depression and pain interference, with the mediating role of pain catastrophizing on those associations among an ethnic minority elderly group with chronic pain. High prevalence of depression ${ }^{13}$ and pain severity ${ }^{9}$ among this particular group of community dwelling Korean immigrants in the US is a known significant factor, and the current study's findings add to the literature by providing evidence of role of pain catastrophizing on the path from depression through chronic pain.

The proportion of elderly suffering from comorbid depression and chronic pain was $45.7 \%$ in the current study; this is greater compared to the previously reported prevalence of this comorbidity in the general elderly population $(13 \%){ }^{29}$ The negative influence of depression on chronic pain outcomes found in this study is consistent with previous studies. Previous studies have reported emotional distress is a risk factor of prognostic indicators for patients with chronic pain, ${ }^{30}$ and patients with depression are more likely to suffer from chronic pain. ${ }^{31}$ In this study, participants with depression expressed higher levels of pain severity and interference for daily life activities compared to those without depression. The overall pain severity scores (3.8 on $0-10$ scale) were somewhat lower in the current study's sample compared to other studies of elderly from Sweden (4.1-4.9 on 0-10 scale) $)^{32}$ and China (50 on $0-100$ scale). ${ }^{16}$ The pain catastrophizing score in the current study was higher compared to that of Chinese elderly (11.2 vs 15.6) ${ }^{16}$ These findings suggest possible crosscultural and intra-racial differences in reporting pain and pain-associated mood, including depression and pain catastrophizing. Specifically, Korean elderly expressed less pain severity, but greater depression and pain catastrophizing. Cultural norms of enduring pain and high pain tolerance may influence these findings, ${ }^{4,7}$ and clinicians should consider these factors when caring for this group of patients.

In this study, we found support for our hypothesis that pain catastrophizing has a mediating effect on the association between depression and pain outcomes. Pain catastrophizing, a maladaptive cognitive response to pain, was higher in those with greater depressive symptoms, and this may have influenced their perception of pain severity, and the levels of interference on activities of daily living 
due to pain. Depressive symptoms did not influence pain severity and pain interference directly, but pain catastrophizing showed indirect effects via helplessness, rumination, and magnification on pain severity and pain interference. This finding is supported by a previous brain imaging study that found pain catastrophizing was related to brain areas involved in pain processing, whereas there was no clear association with depressive symptoms. ${ }^{33}$

Findings of the current study suggest that focusing on reducing pain catastrophizing may be important to improve pain outcomes for elderly individuals with depression. Recently, researchers have developed and tested emotion-focused cognitive interventions to improve pain outcomes for patients with chronic pain with comorbid depression. They reported that focusing on a person's emotion-regulation process and providing cognitive behavioral therapy to improve coping skills were helpful to modify their negative emotion-regulation process, which ultimately resulted in better outcomes related to chronic pain. ${ }^{34}$ Other studies tested the influence of mindfulness ${ }^{35}$ and spiritual well-being ${ }^{36}$ on reducing pain catastrophizing. In addition to the current evidence on interventions for chronic pain, we suggest that treatments or interventions to help elderly engaging in less catastrophic thinking should be developed to better manage chronic pain.

Among the covariates, multisite pain was a significant factor augmenting pain severity and pain interference, controlling for other study variables. Comorbidities were also significantly associated with pain severity, controlling for other variables. These findings are consistent with previous findings reporting significant impacts of multisite pain and comorbid conditions on pain-related outcomes and psychological status. ${ }^{19,37}$ Multisite pain and multimorbidity are common in the elderly, therefore, these factors should be considered to better understand and manage the multi-dimensional aspects of pain in elderly population.

While the study adds significant findings to the current status of science, it has several limitations. First, generalization is limited due to the results coming from a convenience sample from a specific region where a relatively large population of Asian immigrants is residing. Second, we were not able to include chronic pain prognostic variables, such as pain-induced disability, pain-related medication adherence, or physical activity levels. Lastly, variables related to social support or relationships with others (eg, friends, relatives, and children) were not included in this study, which may affect pain experience of immigrants. Future suggested research includes longitudinal studies with larger samples investigating these outcomes and their relation to depression and studies that compare these outcomes among diverse ethnic groups.

\section{Conclusion}

Pain catastrophizing contributes to high levels of pain severity and interference in elderly individuals with comorbid chronic pain and depression. Korean elderly immigrants with chronic pain are highly comorbid with depression, and greater pain severity and interference may be explained by higher levels of pain catastrophizing. To reduce chronic pain disparity, appropriate culturally tailored programs focused on redirecting pain catastrophizing cognitive process should be developed for elderly Asian Americans. A multi-dimensional approach to pain, taking into consideration comorbidities and multisite pain should be considered in chronic pain management in the elderly Asian immigrant population.

\section{Data Sharing Statement}

The author (Hee Jun Kim, hkim20@ajou.ac.kr) will provide data upon reasonable request.

\section{Acknowledgments}

This study was supported by the Faculty Development \& Research Committee Grant (\#15740) from Towson University.

\section{Disclosure}

The authors declare no conflicts of interest for this work.

\section{References}

1. International Association for the Study of Pain. Part III: pain terms, a current list with definitions and notes on usage. In: Merskey $\mathrm{H}$, Bogduk N, editors. Classification of Chronic Pain. 2nd ed. Seattle: IASP Press; 1994:209-214

2. Müller R, Landmann G, Béchir M, et al. Chronic pain, depression and quality of life in individuals with spinal cord injury: mediating role of participation. J Rehabil Med. 2017;49(6):489-496. PMID: 28597908. doi:10.2340/16501977-2241.

3. Turk DC, Fillingim RB, Ohrbach R, Patel KV. Assessment of psychosocial and functional impact of chronic pain. J Pain. 2016;17(9Suppl): T21-49. PMID: 27586830. doi:10.1016/j.jpain.2016.02.006.

4. Kawi J, Reyes AT, Arenas RA. Exploring pain management among asian immigrants with chronic pain: self-management and resilience. J Immigr Minor Health. 2019;21(5):1123-1136. PMID: 30182206. doi:10.1007/s10903-018-0820-8.

5. Andreae MH, White RS, Chen KY, et al. The effect of initiatives to overcome language barriers and improve attendance: a cross-sectional analysis of adherence in an inner city chronic pain clinic. Pain Med. 2017;18(2):265-274. PMID: 28204760; PMCID: PMC6376453. doi: $10.1093 / \mathrm{pm} / \mathrm{pnw} 161$. 
6. Nyen S, Tveit B. Symptoms without disease: exploring experiences of non-western immigrant women living with chronic pain. Health Care Women Int. 2018;39(3):322-342. doi:10.1080/07399332.2017.1370470

7. Kim HJ, Greenspan JD, Ohrbach R, et al. Racial/ethnic differences in experimental pain sensitivity and associated factors - Cardiovascular responsiveness and psychological status. PLoS One. 2019;14(4): e0215534. PMID: 30998733; PMCID: PMC6472780. doi:10.1371/ journal.pone.0215534.

8. Dhatt G, Simpson P, MacDonald V. Self-management of osteoarthritis: a culturally-specific chronic care model for South Asians. Int J Orthop Trauma Nurs. 2012;16(3):121-127. doi:10.1016/j. ijotn.2012.01.001

9. Kim HJ, Chang SJ, Park H, et al. Intra-ethnic differences in chronic pain and the associated factors: an exploratory, comparative design. $J$ Nurs Scholarsh. 2020;52(4):389-396. PMID: 32413245. doi: 10.1111/jnu.12564

10. Doan L, Manders T, Wang J. Neuroplasticity underlying the comorbidity of pain and depression. Neural Plast. 2015;2015:504691. doi:10.1155/2015/504691

11. Walker AK, Kavelaars A, Heijnen CJ, Dantzer R. Neuroinflammation and comorbidity of pain and depression. Pharmacol Rev. 2013;66 (1):80-101. PMID: 24335193; PMCID: PMC3880465. doi:10.1124/ pr.113.008144.

12. Tappe-Theodor A, Kuner R. A common ground for pain and depression. Nat Neurosci. 2019;22(10):1612-1614. PMID: 31455879. doi:10.1038/s41593-019-0499-8.

13. Kim HJ, Park E, Storr CL, Tran K, Juon HS. Depression among Asian-American adults in the community: systematic review and meta-analysis. PLoS One. 2015;10(6):e0127760. PMID: 26029911; PMCID: PMC4452590. doi:10.1371/journal.pone.0127760.

14. Rusu AC, Gajsar H, Schlüter MC, Bremer YI. Cognitive biases toward pain: implications for a neurocognitive processing perspective in chronic pain and its interaction with depression. Clin J Pain. 2019;35(3):252-260. PMID: 30499835. doi:10.1097/ AJP.0000000000000674.

15. Dong HJ, Gerdle B, Bernfort L, Lå L, Dragioti E. Pain catastrophizing in older adults with chronic pain: the mediator effect of mood using a path analysis approach. J Clin Med. 2020;9(7):2073. PMID: 32630330; PMCID: PMC7408783. doi:10.3390/jcm9072073.

16. Cheng ST, Leung CMC, Chan $\mathrm{KL}$, et al. The relationship of self-efficacy to catastrophizing and depressive symptoms in community-dwelling older adults with chronic pain: a moderated mediation model. PLoS One. 2018;13(9):e0203964. PMID: 30226892; PMCID: PMC6143242. doi:10.1371/journal. pone.0203964.

17. Wood BM, Nicholas MK, Blyth F, Asghari A, Gibson S. The mediating role of catastrophizing in the relationship between pain intensity and depressed mood in older adults with persistent pain: a longitudinal analysis. Scand J Pain. PMID: 28850461. 2016;11:157-162. doi:10.1016/j.sjpain.2015.12.009

18. Wood BM, Nicholas MK, Blyth F, Asghari A, Gibson S. Catastrophizing mediates the relationship between pain intensity and depressed mood in older adults with persistent pain. J Pain. 2013;14 (2):149-157. PMID: 23265846. doi:10.1016/j.jpain.2012.10.011.

19. Butera KA, Roff SR, Buford TW, Cruz-Almeida Y. The impact of multisite pain on functional outcomes in older adults: biopsychosocial considerations. J Pain Res. 2019;12:1115-1125. PMID: 30992680; PMCID: PMC6445225. doi:10.2147/JPR.S192755.

20. Cleeland CS. The Brief Pain Inventory, user guide. Houston, TX: Author; 1991. Available from https://www.mdanderson.org/docu ments/Departments-and-Divisions/Symptom-Research/BPI_ UserGuide.pdf. Accessed March 2, 2021.

21. Yun YH, Mendoza TR, Heo DS, et al. Development of a cancer pain assessment tool in Korea: a validation study of a Korean version of the brief pain inventory. Oncology. 2004;66(6):439-444. doi:10.1159/ 000079497
22. Donnelly PL, Kim KS. The patient health questionnaire (PHQ-9K) to screen for depressive disorders among immigrant Korean American elderly. J Cult Divers. 2008;15(1):24-29.

23. American Psychiatric Association. Diagnostic and Statistical Manual of Mental Disorders. 5th ed. Washington, DC: American Psychiatric Association; 2013.

24. Shin J, Park S, Cho S, et al. Validation of patient health questionnaire-9 Korean version (PHQ-9K) scale for screening depression among Korean Americans in community settings. $J$ Theory Constr Test. 2010;14(2):45-50.

25. Sullivan MJL, Bishop SR, Pivik J. The pain catastrophizing scale: development and validation. Psychol Assess. 1995;7(4):524-532. doi:10.1037/1040-3590.7.4.524

26. Goodin BR, Kronfli T, King CD, et al. Testing the relation between dispositional optimism and conditioned pain modulation: does ethnicity matter? J Behav Med. 2013;36(2):165-174. doi:10.1007/s10865012-9411-7

27. Cho S, Kim H, Lee J. Validation of the Korean version of the pain catastrophizing scale in patients with chronic non-cancer pain. Qual Life Res. 2013;22(22):1767-1772. doi:10.1007/s11136-012-0308-2

28. Hayes AF, Scharkow M. The relative trustworthiness of inferential tests of the indirect effect in statistical mediation analysis: does method really matter? Psychol Sci. 2013;24(10):1918-1927. PMID: 23955356. doi:10.1177/0956797613480187.

29. Zis P, Daskalaki A, Bountouni I, Sykioti P, Varrassi G, Paladini A. Depression and chronic pain in the elderly: links and management challenges. Clin Interv Aging. 2017;12:709-720. PMID: 28461745; PMCID: PMC5407450. doi:10.2147/CIA.S113576.

30. Tseli E, Vixner L, LoMartire R, et al. Prognostic factors for improved physical and emotional functioning one year after interdisciplinary rehabilitation in patients with chronic pain: results from a national quality registry in Sweden. J Rehabil Med. 2020;52(2):jrm00019. PMID: 31995224. doi:10.2340/16501977-2648.

31. Ohayon MM, Stingl JC. Prevalence and comorbidity of chronic pain in the German general population. J Psychiatr Res. 2012;46 (4):444-450. $\quad$ PMID: 22265888. doi:10.1016/j. jpsychires.2012.01.001.

32. Denkinger MD, Lukas A, Nikolaus T, Peter R, Franke S, ActiFE study group. Multisite pain, pain frequency and pain severity are associated with depression in older adults: results from the ActiFE Ulm study. Age Ageing. 2014;43(4):510-514. PMID: 24603284. doi:10.1093/ageing/afu013

33. Malfliet A, Coppieters I, Van Wilgen P, et al. Brain changes associated with cognitive and emotional factors in chronic pain: a systematic review. Eur J Pain. 2017;21(5):769-786. PMID: 28146315. doi: 10.1002/ejp.1003

34. Boersma K, Södermark M, Hesser H, et al. Efficacy of a transdiagnostic emotion-focused exposure treatment for chronic pain patients with comorbid anxiety and depression: a randomized controlled trial. Pain. 2019;160(8):1708-1718. PMID: 31335641; PMCID: PMC6687409. doi:10.1097/j.pain.0000000000001575.

35. Conti Y, Vatine JJ, Levy S, et al. Pain catastrophizing mediates the association between mindfulness and psychological distress in chronic pain syndrome. Pain Pract. 2020;20(7):714-723. PMID: 32285576. doi: 10.1111/papr.12899

36. Shaygan M, Shayegan L. Understanding the relationship between spiritual well-being and depression in chronic pain patients: the mediating role of pain catastrophizing. Pain Manag Nurs. 2019;20 (4):358-364. PMID: 31103504. doi:10.1016/j.pmn.2018.12.001

37. van Hecke O, Hocking LJ, Torrance N, et al. Chronic pain, depression and cardiovascular disease linked through a shared genetic predisposition: analysis of a family-based cohort and twin study. PLoS One. 2017;12(2):e0170653. PMID: 28225781; PMCID: PMC5321424. doi:10.1371/journal.pone.0170653. 


\section{Publish your work in this journal}

The Journal of Pain Research is an international, peer reviewed, open access, online journal that welcomes laboratory and clinical findings in the fields of pain research and the prevention and management of pain. Original research, reviews, symposium reports, hypothesis formation and commentaries are all considered for publication. The manuscrip

management system is completely online and includes a very quick and fair peer-review system, which is all easy to use. Visit http:// www.dovepress.com/testimonials.php to read real quotes from published authors. 